\title{
Optimizing MPBSM Resource Allocation Based on Revenue Management: A China Mobile Sichuan Case
}

\author{
Xu Chen \\ School of Management and Economics, University of Electronic Science and Technology of China, Chengdu 611731, China \\ Correspondence should be addressed to Xu Chen; xchenxchen@263.net
}

Received 13 January 2015; Accepted 9 April 2015

Academic Editor: Peter Jung

Copyright ( $) 2015 \mathrm{Xu}$ Chen. This is an open access article distributed under the Creative Commons Attribution License, which permits unrestricted use, distribution, and reproduction in any medium, provided the original work is properly cited.

\begin{abstract}
The key to determining the network service level of telecom operators is resource allocation for mobile phone base station maintenance (MPBSM). Given intense market competition and higher consumer requirements for network service levels, an increasing proportion of resources have been allocated to MPBSM. Maintenance costs account for the rising fraction of direct costs, and the management of MPBSM resource allocation presents special challenges to telecom operators. China Mobile is the largest telecom operator in the world. Its subsidiary, China Mobile Sichuan, is the first in China to use revenue management in improving MPBSM resource allocation. On the basis of comprehensive revenue (including both economic revenue and social revenue), the subsidiary established a classification model of its base stations. The model scientifically classifies more than 25,000 base stations according to comprehensive revenue. China Mobile Sichuan also conducted differentiation allocation of MPBSM resources on the basis of the classification results. Furthermore, it optimized the assessment system of the telecom base stations to establish an assurance system for the use of MPBSM resources. After half-year implementation, the cell availability of both VIP base stations and total base stations significantly improved. The optimization also reduced economic losses to RMB 10.134 million, and enhanced customer satisfaction with network service by $3.2 \%$.
\end{abstract}

\section{Introduction}

In May 2008, the Chinese government announced the restructuring of the Chinese telecom industry. The restructured China Mobile, China Telecom, and China Unicom obtained 3G licenses for TD-SCDMA, CDMA2000, and WCDMA coverage, respectively [1]. The restructuring and issuance of operating licenses enabled the telecom operators to provide comprehensive services without policy restrictions. Operators who have previously been restricted to providing mobile services can now offer fixed network services and vice versa. The restructuring ushered the Chinese telecom industry into an era of comprehensive service competition [2]. This development has facilitated the economic globalization of telecommunications in China and enabled Chinese telecom operators to satisfy the diverse demands of their users and respond to the trends of the world telecom industry. The world's top 10 telecom operators currently implement comprehensive service operation, which translates to more service choices for consumers. Such operation has restored the competitive balance between mobile and fixed network operators in the Chinese telecom market. It has prompted more vigorous competition among providers because of the higher standards imposed on telecommunications services.

The core service of telecom operators is network service, and the foundation of network service is the base station. Network services are severely affected or disrupted by operational problems such as power failure and equipment malfunctions. Thus, the maintenance of telecom base stations is key to network service competition. The level of mobile phone base station maintenance (hereafter referred to as MPBSM) depends largely on the systematic allocation of resources. Resource allocation for MPBSM is a prevalent problem of Chinese and international telecom operators. MPBSM resources are typically characterized by the following features. First, the resources are of diverse types, covering items such as vehicles, instruments and meters, oil engines, raw materials, and spare parts, as well as manpower requirements including maintenance engineers, network optimization engineers, staff training, and technical support. Second, MPBSM resources use 
up a large amount of capital. According to a survey, the maintenance costs incurred by Chinese telecom operators account for about $10 \%$ of revenue and $30 \%$ of direct costs. The current lack of systematic and scientific methods for MPBSM resource allocation emphasizes the theoretical and practical value of strengthened research in this area.

The US airline industry introduced revenue management in the 1980s [3]. Other industries such as the hotel [4], restaurant [5], tourism [6], and hi-tech manufacturing sectors [7] followed suit. The foundation of revenue management is market segmentation, and the primary measures for segmentation are pricing and resource allocation. With the continuous development of the telecom industry, an increasing number of researchers began to focus on revenue management applications. Humair [8], who was one of the earliest researchers in this area, proposed three levels from which telecom revenue management can be studied: the user service level, network link level, and network traffic level. His research provided the overall framework for telecom revenue management. Thus far, many studies have used pricing as a means of regulating network resources to solve problems such as congestion [9-11]. Existing research on resource allocation mainly focuses on wireless network resource allocation [1216]. None of the aforementioned studies involve resource allocation for MPBSM.

The present study takes China Mobile Sichuan as an example, and we use revenue management in improving the efficiency of MPBSM resource allocation. China Mobile is the world's largest telecom operator, mainly engaged in mobile voice, data, IP telephone, and multimedia services. It has the largest network and number of users in the world. By the end of June 2014, China Mobile will have more than $1,500,000$ base stations and 791 million users [17]. China Mobile Sichuan, a wholly owned subsidiary of China Mobile, has 21 regional branches. By end of 2012, it had more than 25,000 base stations and 50 million users. The Sichuan telecom market has the typical features of a Chinese telecom market and is a microcosm of the world telecom industry. The economic development of Sichuan is unbalanced, with both economically developed and backward areas. For instance, the per capita GDP of Chengdu and Panzhihua is more than US\$ 5,000, whereas that of Bazhong and Guangyuan is less than US\$1,500. East and west Sichuan differ in geographical features. The landform is complex and diverse and is composed of plains, hills, mountains, plateaus, and valleys, among others. These special geographical features pose a huge challenge to China Mobile Sichuan in terms of MPBSM, making it a worthwhile research direction. The results of such investigations are anticipated to be of value to both Chinese and international telecom operators.

The main challenges of research and implementation in this study are as follows.

(1) Base Station Classification. Base stations are classified by revenue, including both economic revenue and social revenue. Economic revenue covers that earned from voice and data services. The issues that require resolution include determining the manner by which a trade-off between different revenue sources may be achieved, ascertaining base station revenue, and identifying the number and proportion of various types of base stations in different areas, given the variations in base station revenue caused by the unbalanced economic development in Sichuan.

(2) Resource Allocation. The types of base stations in different areas vary and each branch has differing amounts of resources. Under this backdrop, we determine how to implement resource allocation that corresponds with the status and development levels of different branches. We also decide on strategies for modifying assessment methods and guaranteeing appropriate use of resources in accordance with the requirements of China Mobile Sichuan. Furthermore, we strengthen the implementation of VIP MPBSM.

To the aforementioned ends, we carry out systematic research and implementation and use the insights we acquire for future recommendations. The rest of the paper is organized as follows. In Section 2, we analyze the methods for base station classification and resource allocation, as well as the problems encountered in such activities. In Section 3, we discuss the formulation of a scientific classification standard, in which economic and social revenues are simultaneously considered. We also develop a management system for classifying the base stations in China Mobile Sichuan. Section 4 presents the influencing factors, weight, and methods of resource allocation. In Section 5, we discuss measures for ensuring that all the branches reasonably use resources by assessment modification. We show the economic and social revenue realized through the implementation of this project. Finally, Section 6 reiterates the main findings of this study.

\section{Previously Applied Methods and Problems Encountered}

Revenue management for MPBSM is rooted in base station classification and maximizes base station revenue through MPBSM resource allocation. In this section, we introduce the previously applied base station classification and resource allocation methods, as well as the problems encountered by China Mobile Sichuan in accomplishing these activities.

2.1. Base Station Classification. Base station classification comprises two parts: the criteria for and number of VIP base stations. The methods previously applied by China Mobile Sichuan are described as follows.

(i) Base Station Classification Criteria. The base stations are classified into VIP and common types according to voice service revenue. With the rapid growth of data services, the proportion of data service revenue and total revenue increased, and data service was gradually transformed from nonmainstream to mainstream service. In January to November 2011, China Mobile Sichuan earned the revenue of RMB 4.46 billion from data services; this figure accounted for $28.48 \%$ of total revenue. GPRS traffic in January 2012 reached 299,700 GB, five times that in January 2011. The original VIP base stations are defined according to voice service revenue, 
without the consideration for data service. In addition to economic revenue, social revenue should also be considered a factor in base station classification. For example, the base stations cover VIP enterprises, governments, commercial zones, transport points, and so forth. Therefore, classifying base stations using voice service revenue as the sole basis is not a rational approach.

(ii) Number of VIP Base Stations. China Mobile Sichuan preset the number of VIP base stations in 21 regional branches at $20 \%$ of its total. Given that the number of VIP base stations is unrelated to network resource allocation and performance assessment, the regional branches upheld largely different standards for VIP base station classification. Thus, the proportion of VIP base stations varied from $2.12 \%$ to $23.48 \%$, indicating that presetting a proportion of VIP base stations is an unreasonable strategy.

The quality of VIP base stations in different branches varied because of the factors mentioned above. For example, the $16.73 \%$ that accounts for the VIP base stations at Chengdu branch earned a VIP base station revenue proportion of $27.56 \%$. For Nanchong branch, however, its base station proportion of $16.63 \%$ earned a revenue of $36.53 \%$.

2.2. Resource Allocation. China Mobile Sichuan has various MPBSM resources. The mother company is the main channel that supplies these resources, but each branch can also input some of its own resources to the MPBSM system. Through screening, we weed out the resources that could not be measured or were difficult to deploy and classify the resources into two dimensions: resource types and the effect of VIP base station proportion on resources. The resources are also divided into fixed and variable resources. Fixed resources pertain to one-time investments that can be used in the long term; these include vehicles and instruments. Variable resources are those that require continuous investment and are rapidly consumed. These include related service fees and perishable goods. On the basis of the effect of VIP base station proportion on resources, resources can be divided into two types, namely, strongly affected and weakly affected resources. The former are those that require more investment as VIP base station proportion increases. Examples are materials and oil engines. Resource allocation comprises two components: the resource allocation method and assurance of efficient resource use. The previously applied methods are described as follows.

(i) Resource Allocation Method. In the past, China Mobile Sichuan allocated its resources according to the number of VIP base stations, a method regarded as unscientific. As a result, resource allocation could not match branch demand. Let us take oil engine as an example. The largest gap between the demand and supply of oil engines was observed at Chengdu branch. The gap amounted to 1131 sets. At the same time, the number of oil engines in some other branches surpassed demand, as observed at Mianyang branch, which had an excess of 227 sets.

(ii) Assurance of Resource Use. Previous assessment systems did not distinguish between VIP and normal base stations and did not highlight the importance of the former. Meanwhile, the lack of an assurance system for resource use caused a deficiency in the guidance scheme intended to steer branches toward strengthened allocation of MPBSM resources to the VIP base stations. These flaws in the system resulted in inefficient resource allocation and use, as well as unmaximized base station revenue. To solve these problems, we conduct research, optimization, and implementation from the perspectives of base station classification and resource allocation.

\section{Base Station Classification}

According to the classic market segments of revenue management, base station classification is based on the revenue earned by a base station. Base station classification is the foundation of scientific resource allocation and the core of MPBSM revenue management. Economic revenue refers to that earned from voice and data services, while social revenue pertains to the efficient coverage of all important operational areas. Effective base station management and operation enhance customer satisfaction and enable the accomplishment of communications dispatch, covering the functions of key transport nodes, transportation hub base stations, and so forth.

We first establish the base station classification model using economic revenue as basis. We calculate the economic revenue (including both voice and data services revenue) and use clustering methods and activity-based classification (ABC) to determine the high economic revenue base stations (HERBS, hereafter). Then, we establish the base station classification model using social revenue as basis. We analyze the factors that influence the social revenue and use the Delphi method to determine classification criteria. Subsequently, we set up the base station classification model on the basis of comprehensive revenue (both economic and social revenue) and classify the base stations into Diamond, Gold, Silver, and Bronze degrees following a descending order from the highest comprehensive revenue to the lowest. On the basis of these completed tasks, we develop the Base Station Classification System for China Mobile Sichuan. This system, which is grounded on revenue management, is used to classify the base stations of China Mobile Sichuan.

3.1. Base Station Classification Model Based on Economic Revenue. The economic revenue of a base station includes both voice and data service revenue. Base stations are classified based on their economic revenue to facilitate the organization of HERBS in a scientific manner. The process involves the following steps. (1) The economic revenue of a base station is calculated. (2) The branches are subjected to cluster analysis on the basis of the economic revenue. (3) Finally, the classification criteria based on the economic revenue are determined.

3.1.1. Calculating the Economic Revenue of Base Stations. Given that the existing systems of China Mobile Sichuan do not enable the direct collection of economic revenue data on the base stations, we propose an innovative method, in which 
economic revenue is used to comprehensively consider voice and data services. The calculation method is implemented as follows. First, we collect monthly data on the base station voice traffic (ERL), data traffic (Mbit), total voice service revenue (Yuan), and total data service revenue (Yuan) of each regional branch. The data collection enables the calculation of the average price of voice service (Yuan/ERL) and data service (Yuan/Mbit) per month of each regional branch. Then, we calculate the voice service revenue per month of each base station according to the voice traffic and average price of voice service per month. The data service revenue is calculated in the same manner. Finally, we add the voice service revenue and data service revenue of each base station to obtain the total revenue of each base station. According to management needs, the data on base station economic revenue should be updated every three months.

\subsubsection{Cluster Analysis of Branches Based on Economic Revenue}

(i) The Need for Cluster Analysis. Because economic development, geographical features, and other factors in each region vary, the revenue of base stations in different regional branches and the corresponding degree of dispersion also differ. Using a uniform proportion of revenue to determine the HERBS in different regional branches results in considerably divergent HERBS numbers. Employing a uniform proportion of numbers to determine the HERBS in different regional branches results in significantly varied HERBS economic revenue. For example, if we set $20 \%$ as the uniform proportion of HERBS, the economic revenue of the HERBS of 21 regional branches varies from $16.2 \%$ to $57.28 \%$. Through data analysis, we find that using a uniform standard to determine HERBS is unscientific and unreasonable, either from the perspective of economic revenue or from the number of base stations. The base stations in every regional branch should be classified according to different standards.

Theoretically, we can determine the number of HERBS for 21 regional branches. However, this approach causes a huge gap in the number of HERBS between the largest and smallest dispersion coefficients. It also makes precalculation and postsystem maintenance excessively difficult and expensive. To solve these problems, we establish the relationship between the number of base stations and dispersion degree on the basis of the analysis of the dispersion degree of the base station economic revenue of all the regional branches. Establishing this relationship enables the setting up of the HERBS proportion for all the regional branches, so that the proportion of HERBS numbers and corresponding economic revenue are similar. This method takes into account the differences in base station classification among different regional branches, thereby avoiding the problems caused by excessive categories.

(ii) Selection and Application of Clustering Method. The indices for measuring discrete degrees are mainly range, variance, standard deviation, standard deviation coefficient, and so forth. The average revenue of the base stations differs because the economic development levels of regional branches also differ. Thus, we choose the standard deviation coefficient to compare the discrete degrees of each regional branch. Clustering methods can be divided into partitioning, hierarchical, density-based, grid-based, and model-based methods. Given that only 21 standard deviation coefficients of regional branches are to be clustered, with input being onedimensional data, we select partitioning methods $(k$-means algorithm) and hierarchical methods (CURE algorithm) for clustering.

First, we calculate the standard deviation coefficients of all the regional branches according to the formula for deriving the standard deviation coefficient (standard deviation coefficient $=$ standard deviation/average revenue). We then use the $k$-means and CURE algorithms, with the help of SPSS 17.0, to carry out the cluster analysis of the 21 regional branches. The results are shown in Table 1.

(iii) Determining the Category Number of Clustering. Determining the number of clustering categories is an inevitable yet difficult task; to date, no satisfactory method has been identified. The threshold, $R$ statistics, pseudo F-Statistics, and pseudo statistics methods are the commonly used techniques for determining the number of clustering categories. Determining the categories in this study is easy because onedimensional data are used as input. $R$ statistics is readily understandable and clearly reflects the number of clustering categories; thus, we choose this method to determine the clustering method and number of clustering categories.

If $T$ is the total sum of squares and $P_{G}$ denotes the error sum of squares, then $R$ statistics can be expressed as $R^{2}=$ $1-P_{G} / T$. A large $R^{2}$ shows that the error sum of squares is large if we determine $G$ clustering categories; that is to say, $G$ is a suitable category number. However, obtaining a larger clustering number results in a smaller error sum of squares and a larger $R^{2}$. Thus, we can determine a suitable $G$ only to yield a sufficiently large $R^{2}$, but $G$ itself is small; with the increase in $G$, the rise in $R^{2}$ amplitude is minimal. The $R^{2}$ values calculated using the $k$-means algorithm are shown in Table 2 .

The comparison of the $R^{2}$ values calculated using the two algorithms shows that, at a category number of more than 4 , the value of $R$ was higher than $96 \%$ and the reduction in amplitude was minimal with the decrease in categories. At a category number of $3, R^{2}$ sharply decreased to below $90 \%$ and the reduction in amplitude was large. Thus, choosing 4 as the category number is a reasonable decision.

For a category number of 4 , the clustering method is chosen for determining $R^{2}$. A larger $R^{2}$ means a better clustering result. Because the clustering result of the $k$-means algorithm is the same as that of the CURE algorithm, we deem the results for this category number acceptable.

3.1.3. Determining the Classification Criteria Based on Economic Revenue. After completing clustering, we determine the criteria for HERBS in each region. In determining the HERBS proportion, we use the proportion of HERBS revenue to reversely derive the proportion of HERBS amount. Reverse derivation is an innovative way of using revenue management and enables the scientific determination of HERBS.

First, we use ABC to determine the proportion of the HERBS numbers. We listed all the base stations in China 
TABLE 1: Results of clustering.

\begin{tabular}{|c|c|c|c|c|c|c|c|c|c|c|c|}
\hline \multirow{2}{*}{ Regional branches } & \multirow{2}{*}{$\begin{array}{c}\text { Coefficient of } \\
\text { standard deviation }\end{array}$} & \multicolumn{5}{|c|}{ Results of $k$-means (categories) } & \multicolumn{5}{|c|}{ Results of CURE (categories) } \\
\hline & & 2 & 3 & 4 & 5 & 6 & 2 & 3 & 4 & 5 & 6 \\
\hline Ganzi & 2.0374 & & 1 & 1 & 1 & 1 & & 1 & 1 & 1 & 1 \\
\hline Aba & 1.7268 & 1 & & 2 & 2 & 2 & 1 & 2 & 2 & 2 & 2 \\
\hline Liangshan & 1.6706 & & & & & & & & 2 & 2 & 2 \\
\hline Bazhong & 1.4356 & & 2 & & & & & & & & \\
\hline Ya'an & 1.4004 & & & & & 3 & & & & & 3 \\
\hline Suining & 1.3752 & & & & & & & & & & \\
\hline Nanchong & 1.3166 & & & & 3 & & & & & & \\
\hline Guangyuan & 1.3071 & & & 3 & & & & & 3 & 3 & \\
\hline Dazhou & 1.2806 & & & & & 4 & & & & & 4 \\
\hline Guang'an & 1.2752 & & & & & & & & & & 4 \\
\hline Panzhihua & 1.2353 & & & & & & & & & & \\
\hline Mianyang & 1.1958 & 2 & & & & & 2 & 3 & & & \\
\hline Meishan & 1.1694 & 2 & & & & & 2 & 3 & & & \\
\hline Zigong & 1.1551 & & 3 & & 4 & & & & & & \\
\hline Chengdu & 1.1418 & & & & 4 & 5 & & & & 4 & 5 \\
\hline Leshan & 1.1362 & & & & & & & & & 4 & \\
\hline Luzhou & 1.1025 & & & 4 & & & & & 4 & & \\
\hline Ziyang & 1.0707 & & & & & & & & & & \\
\hline Deyang & 0.9872 & & & & & & & & & & \\
\hline Yibin & 0.9709 & & & & 5 & 6 & & & & 5 & 6 \\
\hline Neijiang & 0.952 & & & & & & & & & & \\
\hline
\end{tabular}

TABLE 2: Calculation results of statistics $R^{2}$ (categories).

\begin{tabular}{lcccccc}
\hline \multicolumn{2}{c}{$T=1.4164$} & & 2 & 3 & 4 & 5 \\
\hline \multirow{2}{*}{$k$-means } & $P_{G}$ & 0.4383 & 0.3092 & 0.1084 & 0.0441 & 0.0185 \\
& $R$ & $83.10 \%$ & $88.41 \%$ & $96.10 \%$ & $98.43 \%$ & $99.34 \%$ \\
\hline \multirow{2}{*}{ CURE } & $P_{G}$ & 0.0210 & 0.0576 & 0.1083 & 0.3619 & 0.4383 \\
& $R$ & $83.10 \%$ & $86.29 \%$ & $96.10 \%$ & $97.94 \%$ & $99.25 \%$ \\
\hline
\end{tabular}

Mobile Sichuan according to the highest economic revenue to the lowest and chose the cumulative economic revenue percentage. We then determine the percentage of the HERBS numbers. We choose $60 \%$ in this study; the corresponding proportion of base station number is $18.29 \%$; that is to say, the benchmark of HERBS percentage is $18.29 \%$ for China Mobile Sichuan. After this, we adjust the benchmark and then determine the proportion of HERBS for each region. The adjustment method is implemented in accordance with the clustering center of each category of regional branches. We calculate the adjustment coefficient (category adjustment coefficient $=$ category inner coefficient of standard deviation/average coefficient of standard deviation of total) and obtain the proportion of HERBS for each region (category proportion of HERBS amount = benchmarking of HERBS amount percentage/category adjustment coefficient). The results are shown in Table 3.

After determining the classification criteria based on economic revenue, we classify all the base stations in each region from the highest- to the lowest-earning stations, thereby allowing for the easy determination of HERBS.
3.2. Base Station Classification Model Based on Social Revenue. Base station classification based on social revenue depends on the importance of the covered areas. The classification process involves the following steps. First, the influencing factors of base station social revenue are analyzed. Second, the importance of these factors is evaluated. Finally, the base stations are classified on the basis of social revenue.

(i) Analyzing the Influencing Factors of Base Station Social Revenue. Through interviews with experts, we gathered 18 characteristics of high social revenue base stations. The base stations are important transmission nodes; cover the office locations of governments and the military; cover airports, railway stations, bus stations, terminals, and other important transportation hubs; bring high economic revenue; cover important customers of the Group; are super stations; cover the locations of meteorology, electricity, gas and water supply command, and control centers; cover the office locations of mobile companies; cover important urban trunk roads; cover important schools; are the main business halls of mobile companies; cover major tourist spots; are built together with 
TABLE 3: Recommended value of proportion of HERBS for each region.

\begin{tabular}{llccc}
\hline Category & Regional branches & Clustering center & Adjustment coefficient & Recommended proportion \\
\hline 1 & Ganzi & 2.0374 & 1.5880 & $11.52 \%$ \\
\hline 2 & Aba, Liangshan & 1.6987 & 1.3241 & $13.82 \%$ \\
\hline 3 & $\begin{array}{l}\text { Bazhong, Yałan, Suining, Nanchong, } \\
\text { Guangyuan, Dazhou, Guang’an, } \\
\text { Panzhihua, and Mianyang }\end{array}$ & 1.3135 & 1.0238 & $17.87 \%$ \\
\hline & $\begin{array}{l}\text { Meishan, Zigong, Chengdu, Leshan, } \\
\text { Luzhou, Ziyang, Deyang, Yibin, and } \\
\text { Neijiang }\end{array}$ & 1.0762 & 0.8388 & $21.81 \%$ \\
\hline
\end{tabular}

TABLE 4: Statistical results of survey on the importance of base stations.

\begin{tabular}{llc}
\hline Type & Reasons that make VIP base stations important & Score \\
\hline 1 & Base stations are important transmission nodes & 4.8 \\
2 & Base stations cover the office locations of governments and the military & 4.7 \\
3 & Base stations cover airports, railway stations, bus stations, terminals, and other important transportation hubs & 4.4 \\
4 & Base stations generate high economic revenue & 4.3 \\
5 & Base stations cover important customers of the Group & 4.2 \\
6 & Base stations are super stations & 4.2 \\
7 & Base stations cover the locations of meteorology, electricity, gas and water supply command, and control centers \\
8 & Base stations cover the office locations of mobile companies \\
9 & Base stations cover important urban trunk roads \\
10 & Base stations cover important schools \\
11 & Base stations are the main business halls of mobile companies \\
12 & Base stations cover major tourist spots \\
13 & Base stations are built together with an 800 M cluster system \\
14 & Base stations are built together with short-wave stations \\
15 & Base stations cover highways and state roads & 4.1 \\
16 & Base stations are ordinary transfer nodes & 3.9 \\
17 & Base stations are built together with 3G base stations \\
18 & Base stations are extremely hard-hit base stations (except for super base stations) & 3.8 \\
\hline
\end{tabular}

an $800 \mathrm{M}$ cluster system; are built together with short-wave stations; cover highways and state roads; are ordinary transfer nodes; are built together with $3 \mathrm{G}$ base stations; and are located in disaster-stricken areas (except for the super base stations).

(ii) Evaluating the Importance of the Influencing Factors. We use the Delphi method to determine the importance of the influencing factors. The questionnaire, designed according to the factors mentioned above, consists of 18 closed questions and two open questions. The answers are hierarchical, with six grades (0: not considered; 1 : very unimportant; 2 : unimportant; 3: neutral; 4 : important; and 5 : very important) to describe the characteristics of a certain base level of social revenue.

We distributed 11 questionnaires to relevant experts from the departments of the networks in China Mobile Sichuan and its regional branches. We collected 10 valid ones. Among these, seven were answered by experts from the mother company. In the regional branches, we asked one expert each from the Chengdu, Deyang, Neijiang, and Ganzi branches to complete the questionnaire. The statistical results are shown in Table 4. (iii) Classifying the Base Stations Based on Social Revenue. On the basis of social revenue, we divide the base stations into three types, namely, high social revenue base station (HSRBS), comparatively high social revenue base station (CHSRBS), and normal social revenue base station. HSRBS refers to the first three types in Table 4, and CHSRBS refers to types 5 to 15 .

3.3. Base Station Classification Model Based on Comprehensive Revenue. Analyzing all the base stations in China Mobile Sichuan using the dimensions of economic and social revenue as bases, we divide the base stations into four categories: Diamond base stations, Gold base stations, Silver base stations, and Bronze base stations. The Diamond and Gold base stations are called VIP base stations. The classification results are shown in Figure 1.

Diamond base stations pertain to the base stations covering important transmission nodes, office locations of governments, the military, and airports, as well as railway stations, bus stations, terminals, and other important transportation hubs. We do not consider the economic revenue of these stations. Gold base stations are HERBS. Silver base stations 
TABLE 5: Results of base station classification in China Mobile Sichuan.

\begin{tabular}{lccc}
\hline Category of base stations & Proportion of amount & Proportion of revenue & Economic contribution \\
\hline Diamond & $12.79 \%$ & $25.07 \%$ & $196.01 \%$ \\
Gold & $13.43 \%$ & $39.21 \%$ & $291.96 \%$ \\
Silver & $11.97 \%$ & $7.65 \%$ & $63.91 \%$ \\
Bronze & $61.82 \%$ & $28.07 \%$ & $45.41 \%$ \\
\hline
\end{tabular}

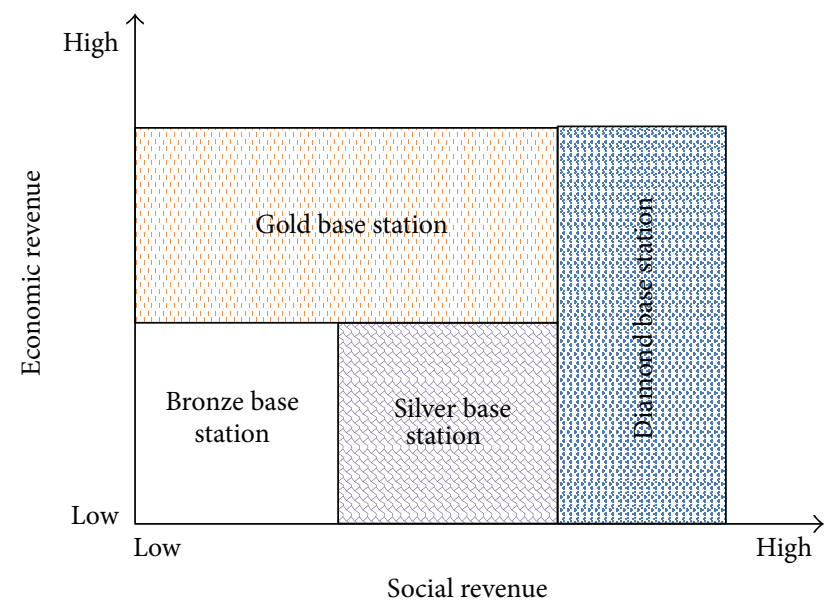

FIGURE 1: Base stations classification.

refer to the low economic revenue but high social revenue base stations. In the Delphi method, 14 types of base stations scored lower than did HERBS and the rest are categorized as Bronze base stations.

3.4. Management System for Base Station Classification of China Mobile Sichuan. China Mobile Sichuan has more than 25,000 base stations, and each of them is heavily loaded with information and computation. To reduce repeated work and improve computational speed, we use the SQL2005 database on the C\#.net platform to develop and implement a management system for the base station classification of China Mobile Sichuan. This system is equipped with a user login module, the original data import module, base station classification module, statistics, and query module.

The management system is open and compatible and can achieve the input of Txt and Excel formats, as well as the output of Excel format. Matching the existing market management, business systems, and office automation systems is easy because of the advantages of user-friendliness, power, ease of use, safety, and reliability.

\subsection{Results of Base Station Classification in China Mobile} Sichuan. Using the system mentioned in the previous section, we classify the base stations in China Mobile Sichuan. Table 5 shows the results.

According to the table, Diamond base stations account for $12.79 \%$ of all the base stations of China Mobile Sichuan. Gold base stations account for 13.43\%; Silver base stations, 11.97\%; and Bronze base stations, $61.82 \%$. The proportions of the number of Diamond, Gold, and Silver base stations are close to one another, whereas the number of Bronze base stations accounts for more than $50 \%$. The comparison of these proportions shows that the proposed program distinguished different base stations and maintained a reasonable proportion. It can therefore serve as a foundation of base station differentiation.

According to the results of base stations classification, the overall revenue for Diamond base stations accounted for $25.07 \%$, Gold base stations accounted for $39.21 \%$, Silver base stations accounted for $7.65 \%$, and Bronze base stations accounted for $28.07 \%$. The number of Gold base stations accounted for $13.43 \%$ but yielded $39.21 \%$ of total revenue, indicating that base stations of this type make a bigger contribution to the economic revenue of China Mobile Sichuan. The proposed method of classification enabled the organization of base stations with high contributions and realized differentiated assurance accordingly.

By comprehensively analyzing the proportion of number and revenue of each type of base station, we determine the degree of economic contribution as follows: Diamond base stations, 196.01\%; Gold base stations, 291.96\%; Silver base stations, $63.91 \%$; and Bronze base stations, $45.41 \%$. The degree of economic contribution of Gold base stations is up to about $292 \%$, indicating that this type of base station makes greater contribution to the economic revenue of China Mobile Sichuan. The proposed classification method enabled the organization of base stations with high contributions and realized differentiated guarantee accordingly. The economic contributions of these stations considerably add to both economic revenue and social revenue.

\section{Resource Allocation}

Corresponding to the capacity allocation of traditional revenue management, resource allocation pertains to the distribution of different types of MPBSM resources to different types of base stations through scientific methods to maximize revenue. Resource allocation is key to achieving the effective management of MPBSM resources and, accordingly, the core of revenue management for such resources. Resource allocation covers three aspects. First, the factors and weights that affect resource allocation are determined. Second, the average and advanced levels of resource allocation on the whole are calculated on the basis of the status of the maintenance resources of each branch. Finally, the resources are allocated on the basis of revenue management.

4.1. Determining Factors and Weights. Through research and interviews, the main factors that affect MPBSM resource allocation are determined to be base station value 
TABLE 6: Weights of base stations determined using the Delphi method.

\begin{tabular}{lccc}
\hline Type of base stations & Diamond base station & Gold base station & Silver base station \\
\hline Average weight & 4.63 & 4.40 & 3.69 \\
\hline
\end{tabular}

TABLE 7: Weights coefficients of all types of base stations.

\begin{tabular}{lcccc}
\hline Type of base stations & Diamond base station & Gold base station & Silver base station & Bronze base station \\
\hline Weight coefficient & 3.08 & 2.92 & 2.45 & 1 \\
\hline
\end{tabular}

(the proportion of HERBS numbers, average revenue of base station, etc.), base station size (the total number of base stations, acreage, time to station, etc.), and special factors (power failure, geographical conditions, etc.).

According to the effects of these factors on different resources, we list the specific factors that affect each type of resource. The project team visited the Department of Networks, Network Optimization Centers, and Network Management Center of China Mobile Sichuan. The team also interviewed 18 relevant experts and conducted a survey. Using the analytical hierarchy process, we obtained the evaluation matrix for the significance of the influencing factors of a variety of resources. The questionnaire that did not satisfy the consistency test was excluded. Then, the team computed the mean of the weight of the remaining questionnaires and obtained the weight of the influence of various types of factors for resource allocation.

4.2. Calculating the Level of Resource Allocation. According to the current resource allocation system of each branch, we calculate the average and advanced levels of MPBSM resource allocation of China Mobile Sichuan. The average level is developed on the basis of the average allocation of certain resources in China Mobile Sichuan. The advanced level allocation is evaluated on the basis of the resource situation at the top three regional branches.

4.3. Method for Resource Allocation Based on Revenue Management. Given the limitations in MPBSM resources, the principle of the China Mobile Sichuan MPBSM resource allocation based on revenue management lies in ensuring the implementation of average allocation standards and encouraging the realization of advanced allocation standards.

The branches, whose levels are lower than the average, must invest according to the suggested standard, with the aid of the mother company. For example, the mother company and its branches invest in network resources at a ratio of $2: 1$. That is, the mother company inputs two units of network resources, while the regional branch invests 1 unit to immediately realize the short-board effects of network services.

The branches whose levels are above average should play a major role in investment while the mother company invests at a certain ratio. For example, at a ratio of $1: 2$, the branch inputs 2 units of resources and the mother company inputs only 1 unit. This approach makes the branches more active and capable of investing in their own resource allocation.

\section{Implementation}

China Mobile Sichuan has 21 regional branches throughout the province and has more than 25000 base stations. For 2012, the investment infused into MPBSM was estimated at RMB 2 billion. Despite this projection, only three engineers are responsible for daily maintenance at the Networks Department of China Mobile Sichuan. The challenge lies in guiding the branches to use the resources from the perspective of revenue management. The effectiveness of implementation is an important index for testing the project and a foundation for subsequent improvement.

5.1. Assurance Mechanism for Resource Use. Assessment can ensure the use of resources in accordance with revenue management. Therefore, we intended to establish an assurance system for resource usage through the optimization of the assessment system. The previous assessment system focuses only on the quantity instead of the quality of the base stations. By contrast, this study considers the established weights of different types of base stations to achieve differential assessment, including quantitative and qualitative evaluation.

As for the indexes, cell availability is the best aggregative indicator of safeguarding the quality of base stations. It can more accurately measure the maintenance capability of a branch. The design of weight coefficient differentiation involves three steps.

First, using the statistical results of the Delphi method, we establish the weights of Diamond, Gold, and Silver base stations, as shown in Table 6.

Second, we determine the evaluation coefficients of the Diamond base station. In our model, the Bronze base station has the lowest economic and social revenue. Thus, we set the evaluation coefficient of the Bronze base station as 1 , and then take it as the basic index for measuring others. Because the Gold base stations only have economic revenue, we use the formula Average revenue of Gold base station/Average revenue of all base station to measure the relative importance of the Gold base stations against the Bronze base stations and then determine the evaluation coefficients of the former. Through the calculation, we obtain the weight of Gold base station as 2.92 .

Third, on the basis of the first two steps, we calculate the weight coefficient of the base stations. The weight coefficients of all the types of base stations are shown in Table 7.

The optimized evaluation coefficients of cell availability enable the assessment of both the quantity and the quality of base stations. These also reflect resource usage. 
TABLE 8: Comparison of cell availability before and after implementation.

\begin{tabular}{lcc}
\hline & $\begin{array}{c}\text { Cell availability of } \\
\text { VIP base stations }\end{array}$ & $\begin{array}{c}\text { Cell availability of } \\
\text { total base stations }\end{array}$ \\
\hline Dec. 2012 & $99.978 \%$ & $99.786 \%$ \\
Jun. 2013 & $99.985 \%$ & $99.923 \%$ \\
\hline
\end{tabular}

5.2. Effect of Implementation. On the basis of the completion of project 2012, China Mobile Sichuan promoted the comprehensive implementation of the new project within the province. After half a year (Jan. to June 2013), the project brought forth significant effects, as shown in Table 8.

The statistics indicate that the cell availability of VIP base stations (including Diamond and Gold base stations) and that of total base stations were enhanced. The benefits to China Mobile Sichuan were as follows.

(i) From the perspective of economic benefits, six-month implementation reduced losses to RMB 10.3134 million or a loss of RMB 1.689 million per month on average. (Note: Economic profitability $=$ Region's total revenue $\times$ (Cell availability in June $2013-$ Cell availability in Dec. 2012 (adjusted))).

(ii) From the perspective of social benefits, the implementation raises safeguards for governments; enhances the reliability of command systems; strengthens the guarantee covering the base station of traffic hinges, important transport routes, and scenic spots; and promotes the level of major account satisfaction. In the six months of implementation, customer satisfaction with the network service was raised by $3.2 \%$.

\section{Conclusion}

Comprehensive operation, the current trend of the global telecom industry, has intensified the competition among traditional telecom operators. As an essential part of competition, network service depends largely on the level of resource allocation for MPBSM. With increasing investment in the maintenance of telecom base stations, maintenance costs represent an increasing proportion of direct costs. Therefore, resource allocation for MPBSM has become one of the most important challenges for telecom operators.

China Mobile is the world's largest operator with nearly 800 million customers. As its subsidiary, China Mobile Sichuan has encountered problems in resource allocation for MPBSM. These problems include unscientific classification of base stations, unreasonable allocation of resources, and inefficient use of station maintenance, among others. To resolve these problems, China Mobile Sichuan promoted the allocation of MPBSM resources from the perspective of revenue management.

On the basis of scientific classification of base station by comprehensive revenue, China Mobile Sichuan allocated MPBSM resources in a differentiated manner and established an assurance system for resource use. After half-year implementation, practical problems were solved, and the cell availability of VIP base stations and the total base stations improved. It also generated good economic and social revenue. The implementation will be gradually incorporated into the operations of the China Mobile Group. The successful experiment on China Mobile Sichuan for the adoption of a revenue management to allocate MPBSM resources offers a new perspective to the resource management of base stations. The results can serve as reference for other telecom operators around the world.

On the other hand, although revenue management is widely and successfully implemented in the area of airlines, hotels, and travel, its implementation in the allocation of MPBSM resources raises a few new issues. For example, the target of market segmentation is no longer the end-users, as in traditional revenue management, but the base station of telecom companies. The target of resource allocation is no longer airplane seats or hotel rooms, but MPBSM resources. Moreover, China Mobile Sichuan established an assurance system for the use of MPBSM resources through an assessment system in accordance with actual operations. The first successful implementation of revenue management in the allocation of MPBSM resources by China Mobile Sichuan not only expands the application and scope of revenue management, but also deepens the research on revenue management theory. Further studies can focus on how to apply revenue management in other industries and research fields.

\section{Conflict of Interests}

The authors declare that there is no conflict of interests regarding the publication of this paper.

\section{Acknowledgments}

This research is also partially supported by the National Natural Science Foundation of China (no. 71272128, 71432003), Program for New Century Excellent Talents in University (no. NCET-12-0087), Specialized Research Fund for the Doctoral Program of Higher Education (no. 20130185110006), and Youth Foundation for Humanities and Social Sciences of Ministry of Education of China (no. 11YJC630022).

\section{References}

[1] J. Liu, G. Liu, N. Li, and H. Xu, "Dynamics analysis of game and chaotic control in the Chinese fixed broadband telecom market," Discrete Dynamics in Nature and Society, vol. 2014, Article ID 275123, 8 pages, 2014.

[2] X. Chen and X. Wang, "Free or bundled: channel selection decisions under different power structures," Omega, vol. 53, pp. 11-20, 2015.

[3] X. Chen and G. Hao, "Co-opetition alliance models of parallel flights for determining optimal overbooking policies," Mathematical and Computer Modelling, vol. 57, no. 5-6, pp. 1101-1111, 2013.

[4] H. Mei and Z. Zhan, "An analysis of customer room choice model and revenue management practices in the hotel industry," International Journal of Hospitality Management, vol. 33, no. 1, pp. 178-183, 2013. 
[5] F. Guerriero, G. Miglionico, and F. Olivito, "Strategic and operational decisions in restaurant revenue management," European Journal of Operational Research, vol. 237, no. 3, pp. 1119-1132, 2014.

[6] Z. Schwartz, W. Stewart, and E. A. Backlund, "Visitation at capacity-constrained tourism destinations: exploring revenue management at a national park," Tourism Management, vol. 33, no. 3, pp. 500-508, 2012.

[7] X. Chen, A. H. Tai, and Y. Yang, "Optimal production and pricing policies in a combined make-to-order/make-to-stock system," International Journal of Production Research, vol. 52, no. 23, pp. 7027-7045, 2014.

[8] S. Humair, Yield management for telecommunication networks: defining a new landscape [Doctoral dissertation], MIT, Cambridge, Mass, USA, 2002.

[9] S. K. Nair and R. Bapna, "An application of yield management for Internet service providers," Naval Research Logistics, vol. 48, no. 5, pp. 348-362, 2001.

[10] N. Keon and G. Anandalingam, "A new pricing model for competitive telecommunications services using congestion discounts," INFORMS Journal on Computing, vol. 17, no. 2, pp. 248262, 2005.

[11] M. Bouhtou and G. Erbs, "A continuous optimization model for a joint problem of pricing and resource allocation," RAIRO Operations Research, vol. 43, no. 2, pp. 115-143, 2009.

[12] M. Bouhtou, G. Erbs, and M. Minoux, "Joint optimization of pricing and resource allocation in competitive telecommunications networks," Networks, vol. 50, no. 1, pp. 37-49, 2007.

[13] G. Zachariadis and J. A. Barria, "Dynamic pricing and resource allocation using revenue management for multiservice networks," IEEE Transactions on Network and Service Management, vol. 5, no. 4, pp. 215-226, 2008.

[14] Y. Kastro, G. Isıklar, and A. Başar Bener, "Resource allocation in cellular networks based on marketing preferences," Wireless Networks, vol. 16, no. 1, pp. 27-38, 2010.

[15] G. Perakis and G. Roels, "Robust controls for network revenue management," Manufacturing and Service Operations Management, vol. 12, no. 1, pp. 56-76, 2010.

[16] I. Malanchini, M. Cesana, and N. Gatti, "Network selection and resource allocation games for wireless access networks," IEEE Transactions on Mobile Computing, vol. 12, no. 12, pp. 24272440, 2013.

[17] China Mobile, Operation Data, China Mobile, 2014, http://www .chinamobileltd.com/en/ir/operation.php. 

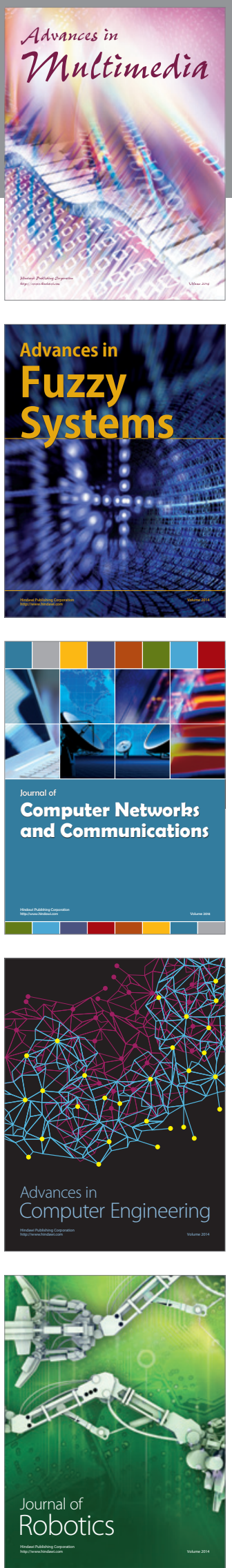

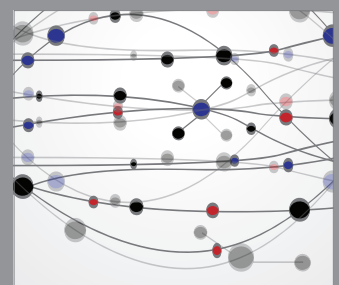

The Scientific World Journal
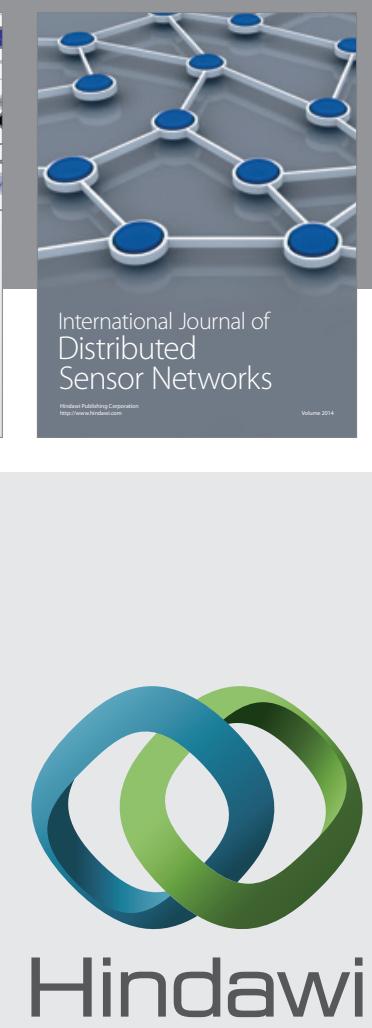

Submit your manuscripts at

http://www.hindawi.com
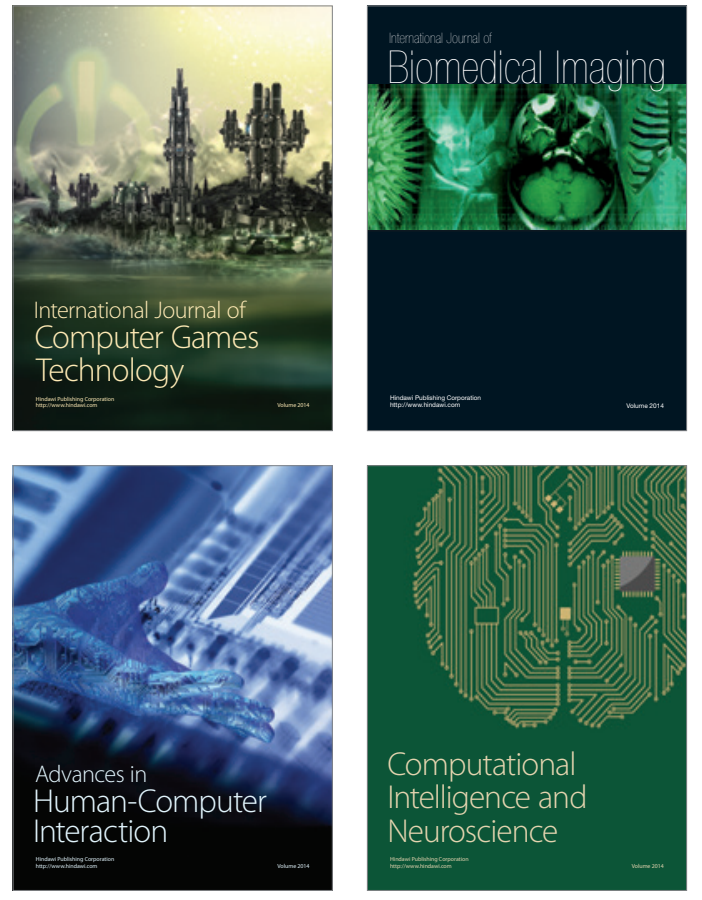
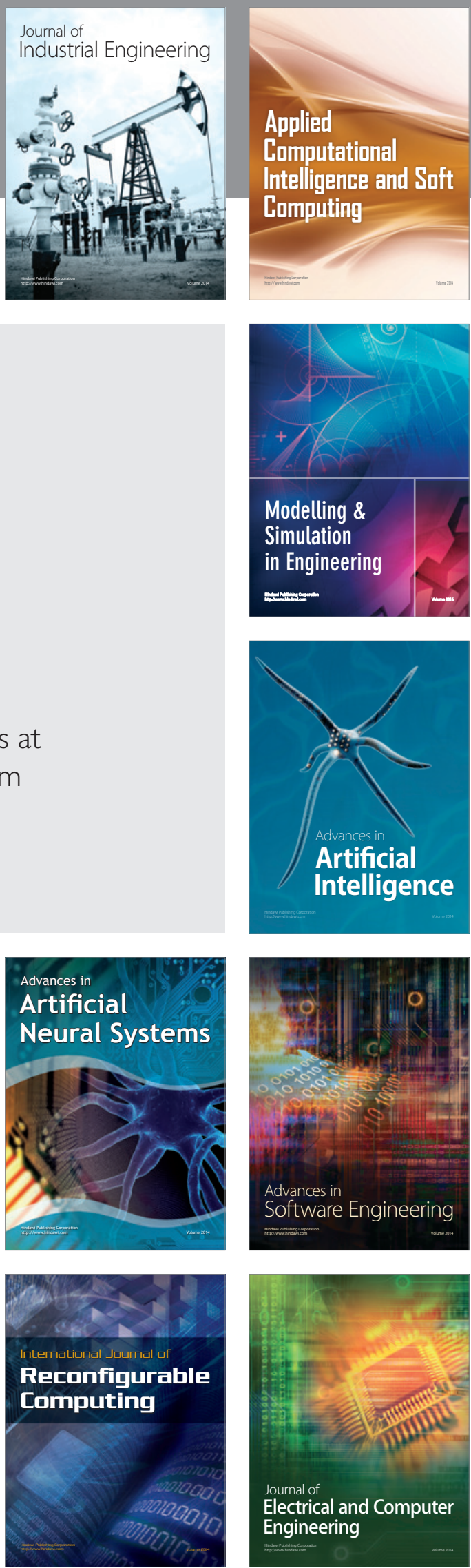